\title{
TEST PERFORMANCE OF CHEST RADIOGRAPHY AS A MEDICAL SURVEILLANCE TECHNIQUE FOR WORKERS EXPOSED TO SILICA DUST
}

Minghui Chen, ${ }^{1}$ Weihong Chen, ${ }^{2}$ Hao Zhang, ${ }^{2}$ Yuewei Liu, ${ }^{2}$ Lap Ah Tse, ${ }^{1}$ Tze Wai Wong ${ }^{1}{ }^{1}$ Chinese University of Hong Kong, NT, Hong Kong; ${ }^{2}$ Huazhong University, Wuhan, China

\subsection{6/oemed-2011-100382.297}

Objectives The purpose of this study is to examine the test performance of periodic chest radiograph for early detection of silicosis by comparing original diagnosis and one panel's evaluation (as reference standard) under different screening frequencies and exposure scenarios and to assess the ability to discriminate of silicosis on chest radiograph.

Methods The study was performed using historical cohort data of workers exposed to silica dust with periodic chest $\mathrm{X}$-ray films in China. Three experienced radiologists were recruited to re-evaluate radiographs according to Diagnostic Criteria of Pneumoconiosis of China. Sensitivity, specificity, positive predictive value, and negative predictive value were calculated to evaluate the agreement between the original and panel's diagnoses. Multivariable models were performed to determine whether statistically significant variation in every test performance measure is associated with different levels of dust exposure index or stage of pneumoconiosis, after adjusting for worker's basic demographic characteristics, smoking data, past and concurrent health problems, and other occupational co-existing concomitants in the workplace. Preliminary

Results Total 356 silicosis patients in the iron ore mine were included, and chest radiographs were 3596 films. The average number of chest radiographs was 10.91 (range: 1-27), and the average interval for patients' chest X-ray screening before and after diagnosis of silicosis was 5.87 and 2.5.

Conclusions We are working on data collection and the data are not sufficient for us to evaluate sensitivity, specificity at this stage. We expect the sensitivity and specificity to be $80 \%$ or more (data will be presented in the conference). 